\title{
Apuntes para reflexionar sobre política científica: el caso de un programa de becas de "vacancia geográfica"
}

\author{
Notes to reflect on scientific policy: the case of a scholarship \\ program of "geographical vacancy"
}

María Itatí Rodríguez itatirodriguez@yahoo.com.ar

http://orcid.org/0000-0002-4299-8003

Instituto de Estudios Sociales y Humanos; Universidad Nacional de Misiones/ Consejo Nacional de Investigaciones Científicas y Técnicas (Argentina)

\section{Resumen}

La política científica contemporánea constituye un rasgo ineludible de las sociedades en las cuales vivimos, convirtiéndose en un capítulo importante de sus agendas. En este contexto, entendemos a las prácticas científicas y tecnológicas como prácticas sociales insertas dentro de determinados contextos políticos, culturales, sociales, económicos, científicos que proponen determinados diálogos y tensiones entre los distintos actores involucrados. En el presente ensayo pretendemos reflexionar sobre la aplicación de una política científica y tecnológica entendida en este contexto como política pública. De esta manera, indagaremos sobre los contextos de producción y enunciación del Convenio de Cooperación entre el Ministerio de Ciencia, Tecnología e Innovación Productiva (MINCyT), el Consejo Nacional de Investigaciones Científicas y Técnicas (CONICET) y la Universidad Nacional de Misiones denominado "Programa de Becas para Áreas de Vacancia Geográfica": ¿qué actores, alianzas, relaciones, dinámicas, tensiones y cristalizaciones institucionales propuso este Convenio de Cooperación? ¿Qué roles ocuparon los distintos actores sociales y políticos en la implementación de esta 
política científico-tecnológica? ¿Qué prioridades y vínculos con otras políticas públicas propuso? ¿Qué aspectos políticos permean las distintas funciones y relaciones sociales de las instituciones universitarias?

Palabras clave: política pública; política científica; ciencia; tecnología.

\section{Abstract}

Contemporary scientific policy constitutes an inescapable feature of the societies in which we live, becoming an important chapter of their agendas. In this context, we understand scientific and technological practices as social practices that are embedded within certain political, cultural, social, economic, and scientific contexts that propose certain dialogues and tensions among the different actors involved. In this essay we intend to reflect on the application of a scientific and technological policy understood in this context as public policy. In this way, we will investigate the contexts of production and enunciation of the Cooperation Agreement between the Ministry of Science, Technology and Productive Innovation (MINCyT), the National Council of Scientific and Technical Research (CONICET) and the National University of Misiones called "Program of Scholarships for Areas of Geographic Vacancy": What institutional actors, alliances, relationships, dynamics, tensions and crystallizations did this Cooperation Agreement propose? What roles did the different social and political actors occupy in the implementation of this scientific-technological policy? What priorities and links to other public policies did you propose? What political aspects permeate the different functions and social relationships of the university institutions?

Keywords: public policy; scientific policy; science; technology.

Una política estatal hace referencia a un conjunto de acciones y omisiones, iniciativas y respuestas (manifiestas o implícitas) en determinado tiempo histórico que manifiestan determinada modalidad de intervención del estado en relación a una cuestión que concita la atención, interés o movilización de otros actores de la sociedad (Oszlak y O’Donell, 1995). En este sentido, se corresponde a una toma de posición que intenta de alguna forma la resolución de una cuestión, que incluye decisiones de una o más organizaciones sociales, simultáneas o 
sucesivas a lo largo del tiempo. La toma de posición no es unívoca, homogénea ni permanente, sino que también atraviesa ambigüedades (Ídem).

En este contexto, una política científica hace referencia a las "medidas colectivas que toma un gobierno para fomentar, de un lado, el desarrollo de la investigación científica y tecnológica y, de otro, a fin de utilizar los resultados de esa investigación para objetivos políticos generales" (Elzinga y Jamison, 1996: 2). Es el "resultado de una interacción dinámica entre actores que representan diferentes culturas políticas" (Ídem).

Las políticas científicas -en diálogo con otras políticas públicas- surgieron en determinado tiempo/espacio de la conformación de los Estados-Nación. Excede a nuestro texto explayarnos en detalles, pero sí consideramos necesario destacar que a pesar de ser un modo de hacer/poder del Estado y sus agentes, han atravesado cambios visibles en las décadas de su formación "como consecuencia de la evolución histórica de la relación de los distintos actores con el poder, pero en gran parte por una mejor comprensión de la naturaleza de la relación ciencia - sociedad y de la eficacia de los diferentes instrumentos de los que se han valido los gobiernos para lograr sus objetivos políticos en relación con la ciencia" (Albornoz, 2007: 58).

De esta manera, la política científica contemporánea constituye un rasgo ineludible de las sociedades en las cuales vivimos, convirtiéndose en un capítulo importante de las agendas públicas y hasta de la preocupación ciudadana como en ningún momento previo de la historia (Ibídem: 48). En el caso de la Argentina, sus transformaciones y aperturas en tanto política científica y tecnológica, son resultado de "configuraciones cambiantes de actores que en distintos momentos fueron capaces de generar diferentes alianzas y provocar confrontaciones en los inestables escenarios de la política y la economía" (Albornoz y Gordon, 2011: 4).

En este contexto, entendemos a las prácticas científicas y tecnológicas como prácticas sociales que se encuentran insertas dentro de determinados contextos políticos, culturales, sociales, económicos, científicos que proponen determinados diálogos y tensiones entre los distintos actores involucrados. En el presente ensayo pretendemos reflexionar sobre la aplicación de una política científica y tecnológica entendida en este contexto como política pública. Para comenzar a reflexionar sobre la política de ciencia y tecnología en nuestros países es necesario indagar sobre las distintas trayectorias de los diversos actores involucrados (Ibídem: 2). En esta oportunidad, nos centraremos en un caso, como propuesta de aproximación a los debates sobre política científica. Advertimos -e invitamos- a que esta discusión continúe mediante el análisis de otros casos, o la incorporación de otras aristas del caso que abordaremos en este caso. 
De esta manera, en el presente ensayo indagaremos sobre el contexto de producción y enunciación del Convenio de Cooperación entre el Ministerio de Ciencia, Tecnología e Innovación Productiva (MINCyT), el Consejo Nacional de Investigaciones Científicas y Técnicas (CONICET) y la Universidad Nacional de Misiones denominado "Programa de Becas para Áreas de Vacancia Geográfica". El mismo tuvo como objetivo general apoyar y fortalecer el Programa de Becas Especiales para las Universidades Nacionales radicadas en las provincias, buscando incentivar el desarrollo de estudios doctorales en Centros de Excelencia localizados en otras partes del país, en graduados provenientes de Universidades situadas en provincias de menor desarrollo. En las distintas convocatorias (desde el 2008 al 2011) se seleccionaron provincias consideradas menos favorecidas en cuanto a la recepción de recursos del Estado (en todas sus formas) para actividades científicas y tecnológicas. En cada uno de estos casos, se había contactado e invitado a la Universidad Nacional que desee participar del presente plan a proponer candidatos para realizar doctorados en centros de excelencia del país, aceptando.

La Universidad Nacional de Misiones (UNaM) -objeto de nuestra discusión- adhiere a dicho Programa en varias convocatorias. Para el presente trabajo se compartirá algunas reflexiones en relación a la última convocatoria de este Programa que se ha concretado que data de 2011. De esta manera, nos interesa comenzar a indagar: ¿qué actores, alianzas, relaciones, dinámicas, tensiones y cristalizaciones institucionales propuso este Convenio de Cooperación? ¿Qué roles ocuparon los distintos actores sociales y políticos en la implementación de esta política científico-tecnológica? ¿Qué prioridades y vínculos con otras políticas públicas propuso? ¿Qué aspectos políticos permean las distintas funciones y relaciones sociales de las instituciones universitarias?

\section{Contextos de producción y enunciación}

En la Argentina, como en la mayoría de los países de América Latina, "las formas institucionales e instrumentales de la política científica tendieron a replicarse miméticamente, siendo frecuentemente imitadas de un país a otro" (Albornoz y Gordon, 2011: 7). Estos países "acomodaron" sus estructuras siguiendo tendencias dominantes para el impulso de la política científica en distintos momentos. Podemos señalar que a finales de la década de 1960 "seis países latinoamericanos crearon consejos de ciencia y tecnología, con diseños y funciones muy similares (Ídem), tal como es el caso de Argentina con la creación del CONACYT y el CONICET. Sin embargo, al interior del Estado argentino, podríamos considerar una tendencia 
que 'benefició' a centros geográficos en la conformación de centros de Investigación + Desarrollo (I+D), dejando en "vacancia" a la mayoría de las provincias argentinas. La implementación de distintos convenios, como es el caso de las Becas CONICET denominadas Área de Vacancia Geográfica, tendría como uno de sus objetivos comenzar a subsanar estos procesos mediante la federalización de determinadas políticas.

De esta manera, la política científica que indagaremos en esta oportunidad se corresponde a un periodo que se inicia en 2003 y que Albornoz y Gordon (2011) lo señalan dividido en dos etapas:

Por un lado, durante el gobierno de Néstor Kirchner, el máximo organismo del sector era el Ministerio de Educación, Ciencia y Tecnología, del que pasó a depender la Secretaría de Ciencia, Tecnología e Innovación Productiva. Esta última puso el énfasis en la planificación a medio y largo plazo, lo cual no dejaba de mostrar un curioso optimismo en un país tan expuesto a las mutaciones abruptas y muchas veces inesperadas. Durante esta gestión se lograron varias metas, como el relanzamiento del CONICET, a la vez que comenzó una etapa de modesta expansión presupuestaria aunque de fuerte incorporación de investigadores y becarios en el CONICET y las universidades, en el contexto de la recuperación y expansión económica experimentada por el país durante el período de gobierno 2003-2007. El gobierno de Cristina Fernández de Kirchner habría de traer como novedad la creación del Ministerio de Ciencia, Tecnología e Innovación Productiva, separado del Ministerio de Educación, del cual depende la Secretaría de Políticas Universitarias. Sobre el alcance real de esta novedad institucional es pronto para emitir un juicio definitivo, aunque ciertos señalamientos iniciales serán formulados en el texto (2011: 11-12).

Desde el inicio del primer periodo que mencionan estos autores, se comenzó a pensar distintas estrategias de mediano plazo en Ciencia, Tecnología e Innovación. En este contexto, se trazaron distintos escenarios, a la vez que se establecieron metas tanto cuantitativas como cualitativas (Albornoz y Gordon, 2011: 31). Un actor clave de estas políticas continuó siendo el CONICET en la medida que fue capaz, "en el marco de su proceso de consolidación institucional, de ampliar significativamente el número de becas para estudios de posgrado otorgadas, y de reabrir el ingreso a la carrera de investigador, que estaba congelado desde mediados de la década de 1990" (Ibídem: 32).

En este contexto, consideramos importante resaltar uno de los objetivos estratégicos de dicho Plan: "aumento de la base científica y de la capacidad tecnológica". De este objetivo se 
desprendieron metas cuantitativas, planes horizontales y criterios de reforma funcional del sistema" (Ibídem: 31). Los planes horizontales se identificaron por cinco componentes principales de un programa de formación de recursos humanos en ciencia y tecnología:

“I) Incorporación de científicos y tecnólogos; II) Formación de investigadores; III) Fortalecimiento de la formación en ingeniería; IV) Radicación de investigadores en el interior del país. Por otra parte, en tanto a la reforma funcional del sistema se plantearon algunos de los siguientes criterios: fortalecimiento de grupos de excelencia en áreas prioritarias mediante la generación de "masa crítica" de investigadores y tecnólogos; atención a la cobertura de áreas de vacancia temática; equilibrio geográfico en la distribución y orientación del sistema, incluyendo la compra de equipamiento y el impulso a programas de modernización tecnológica con criterios de racionalidad regional; aprovechamiento más eficiente y eficaz de los recursos disponibles" (Albornoz y Gordon, 2011: 31).

Es en este contexto de producción y enunciación en el que podemos comenzar a reflexionar sobre el Programa de Becas de Postgrado para Áreas de Vacancia Geográfica (1) creado por el CONICET y el Ministerio de Ciencia, Tecnología e Innovación Productiva de la Nación y que tuvo como objetivo fortalecer las capacidades en recursos humanos de excelencia para actividades de investigación en Universidades Nacionales del interior del país. En este contexto, se invitó a participar a las universidades consideradas como "áreas de vacancia geográfica", entre las cuales estuvo la Universidad Nacional de Misiones. Este tipo de becas compartía las características generales de una Beca de CONICET Tipo I (tres años) con posibilidad de extender a una Tipo II (dos años; ambas se corresponden actualmente a una beca general de cinco años). Sin embargo, podemos destacar algunas de las particularidades de dicho Programa.

Por un lado, los postulantes que obtenían la beca recibirían un estipendio mensual que se corresponde a la ciudad donde se encuentre establecido el lugar de trabajo asignado para la beca. En este caso, al percibir un estipendio correspondiente al lugar asignado, todos centros de investigación consolidados, estos no percibían un ingreso por "zona prioritaria", afectando el ingreso que obtenían cada mes. Esto se podría resarcir con otra cláusula del Programa que dictaba: "durante la beca, la Universidad deberá otorgar o mantener, según corresponda, un cargo docente de dedicación simple con licencia con goce de haberes".

Otra de las cláusulas de esta convocatoria era que luego de completar su formación doctoral y, eventualmente postdoctoral, los becarios y becarias tendrán la obligación de regresar a la 
Universidad que los preseleccionó por un tiempo no menor al que ha usufructuado su beca. En este contexto el programa dictaba que "la Universidad deberá comprometerse a otorgar al becario a su regreso, un cargo docente de dedicación exclusiva". En este contexto, antes del inicio de sus actividades los becarios doctorales del CONICET (2012-2017) firmaron un Compromiso de Regreso en el marco de este Programa. Que en su clausula d) dicta que el becario se compromete una vez finaliza su doctorado "A regresar a la Universidad que lo preseleccionó luego de completar su formación doctoral y, eventualmente, posdoctoral y permanecer en ella durante un tiempo no menor al que ha usufructuado su beca".

Asimismo, la convocatoria acordaba una cláusula de cambio de lugar de residencia durante el período de beca, así como también, "los doctorados propuestos deberán realizarse en una universidad distinta de la que propone al candidato". Esto se realizó en el marco de un proceso de desarraigo en el cual los becarios y becarias cambiaron su lugar de residencia, de grupo de trabajo y de Universidad para insertarse en un nuevo grupo y con un director de beca designado por el CONICET, ubicado en un lugar considerado "centro de excelencia", en relación a número de investigadores, proyectos y recursos económicos.

De esta manera, una vez designado la nómina de nuevos becarios, las Universidades en conjunto con el Ministerio de Ciencia, Tecnología e Innovación Productiva, redactaron un modelo de convenio o adenda. "El mismo deberá ser analizado por el área jurídica de la Universidad con el objeto de realizar las observaciones o modificaciones necesarias para su firma. El convenio establecerá, en todos los casos, la aceptación por las partes firmantes de la aplicación del Reglamento de Becas de Investigación Científica y Tecnológica del CONICET". En el caso de la Universidad Nacional de Misiones, redactó y firmó un convenio que modifica las condiciones de la convocatoria. El mismo fue aprobado por Resolución del Consejo Superior de la UNaM (Nro. 134-12 del 26 de diciembre del 2012), nueve meses después del inicio de actividades de los becarios.

Uno de los puntos que modificó este nuevo convenio es su clausula sexta que dicta "La Universidad, conforme al Régimen de Carrera Docente vigente y en el marco de su Programa de Fortalecimiento Científico-Tecnológico, se compromete a incorporar a su planta docente al BECARIO que haya obtenido su Doctorado"; "La Universidad deberá comprometerse a otorgar al becario a su regreso, un cargo docente de dedicación exclusiva". Podemos destacar dos puntos: por un lado, la Universidad no cumplió con el compromiso de otorgar un cargo de dedicación simple con goce de haberes a los becarios de la convocatoria 2011 al inicio de sus actividades, recortando beneficios a los becarios y becarias que ella misma postuló. En la resolución que aprobó su Consejo Superior menciona que este cargo se generará cuando el 
becario termine su doctorado. Y segundo, en relación al primer punto, hasta la actualidad la Universidad no ha regularizado el cumplimentado de dicho compromiso a la totalidad de los becarios y becarias que han finalizado sus estudios de doctorado. Cabe destacar en el caso de la Universidad Nacional de Misiones, esta no realizó un seguimiento sistematizado de sus becarios doctorales durante los cinco años que duró este convenio, ni se contactó una vez finalizada las mismas para consultar en qué estado de trabajo y retorno estaría cada uno de los becarios. Estuvo en cada becario o becaria acercarse a plantear su situación a los funcionarios de la Universidad para comenzar a reestablecer el vínculo. Tampoco desde el CONICET o el MINCyT hubo un seguimiento o acompañamiento durante los años de beca.

En este contexto, nos parece interesante para continuar con el despliegue de nuestros interrogantes planteados al inicio de este texto, seguir la propuesta de análisis de las cuatro culturas "típicas" que influyen en la formulación de la política científica realizada por Elzinga y Jamison (1996) mediante la cual intentaremos identificar a los distintos actores y procesos en el marco de este Convenio.

En primer lugar, una cultura burocrática que es encarnada en el aparato del estado, que procura administrar y organizar la ciencia para disponerla al servicio de la política. En este contexto podemos observar un interés para poder administrar y organizar un política científicotecnológica que pretende continuar en una línea de trabajo, el de incorporar recursos humanos calificados en provincias con escaso número de doctores. Se destacan las administraciones en gestión de la ciencia que proponen este tipo de iniciativa, las diseña y ejecuta. En este caso, las distintas administraciones pujan en torno a los usos sociales de la ciencia y la tecnología. En este caso, los tres organismos de Estado comprometidos (CONICET, MINCyT y UNaM) se han organizado y firmado un convenio que buscó articular fines y medios de cada uno de los involucrados.

En segundo lugar, la cultura académica encarnada en la comunidad científica, "que busca preservar los valores y la autonomía tradicionales de la ciencia frente a otros intereses" (Albornoz y Gordon, 2011: 4). En este caso, su participación se logró mediante la amplia convocatoria en la cual participaron todas las Unidades Académicas de la universidad, la relevancia de los temas de investigación y las disciplinas. En la convocatoria 2011, la UNaM seleccionó cuarenta becarios doctorales en el marco de dicho convenio, que se distribuyeron con sus lugares de trabajo en distintos puntos del país, especialmente en Ciudad de Buenos Aires, La Plata y Córdoba. Consideramos relevante ofrecer aquí algunos datos sobre sobre la distribución geográfica de los recursos humanos del CONICET del año 2010: 
el 59\% de los recursos humanos del CONICET se concentran en la región bonaerense, el $21 \%$ en la región Centro, y casi un $7 \%$ en la región Cuyo, mientras que el $13 \%$ se localiza en el resto del país. Los datos disponibles para los últimos tres años no indican que se haya cambiado la tendencia, lo que señala la dificultad para revertir la concentración a pesar los dos esfuerzos realizados en el marco de los programas de áreas de vacancia geográfica (Albornoz y Gordon, 2011: 36).

Sin embargo, esta situación reconoció la autonomía de la comunidad científica de la UNaM para formar sus recursos humanos en temas de investigación que se venían desarrollando en la provincia. En este contexto, en el área de Ciencias Sociales y Humanas, cabe señalar en este marco la importancia de la reciente creación del Instituto de Estudios Sociales y Humanos de doble dependencia CONICET-UNaM. En este organismo del sector científico y tecnológico que inició sus actividades a principios de 2015, becarios AVG-CONICET han conformado los primeros recursos humanos y actualmente, como becarios postdoctorales. La creación de dicho Instituto se funda en la necesidad de consolidar en el Nordeste argentino el crecimiento de las Ciencias Humanas y Sociales, con el aporte de investigadores y becarios nucleados para tal fin. Sin embargo, aún no logra promover una planificación concreta que incluya la formación de estos recursos humanos altamente calificados.

En tercer lugar, la cultura económica, representada en los grupos empresarios y los responsables de la política económica, que se interesa por las aplicaciones tecnológicas de la ciencia, orientadas hacia innovaciones rentables. En este sentido, esta cultura involucra a los agentes que están preocupados por los procesos de innovación, específicamente por lo considerado "tecnológico", que deja por fuera lo que se consideraría "científico" o investigación básica. Podemos pensar aquí que la formación de recursos humanos podrían fortalecer proponiendo políticas económicas específicas- un crecimiento en determinados rubros dentro de la provincia. Esto debería estar representado "en los empresarios y los responsables de la política económica, que se interesa por las aplicaciones tecnológicas de la ciencia, orientadas hacia innovaciones rentables" (Albornoz, 2007: 62). Sin embargo, esto no fue pensado en conjunto con este Programa de Becas.

Finalmente, una cultura cívica encarnada en los movimientos sociales que presta atención a las repercusiones sociales de la ciencia (Albornoz y Gordon, 2011). Esta tiene como objetivo pensar el impacto que tiene esta política científico-tecnológica. En este caso, se podría rastrear la participación de estos recursos humanos en el espacio público. En este caso, como parte de esta política tampoco se consideró la conformación de "instituciones que faciliten la participación pública en la política de ciencia y tecnología" (Albornoz, 2007: 62). En dicha 
convocatoria no se ha conformado una "agenda social" que oriente las temáticas y las acciones.

\section{A modo de cierre}

La política científica está instalada en la arena de las relaciones entre la ciencia, el poder, y las demandas sociales. Consideramos necesario pensar estas políticas transversalmente, teniendo en cuenta los procesos culturales, económicos, políticos, etcétera. Los espacios en los cuales se conciben no son neutros, y aún en aparente aplicación, generan disputas que van más allá de las reglamentaciones, convenios y programas. Asimismo, involucran las subjetividades de las personas involucradas, sus trayectorias, expectativas, desafíos.

De esta manera, la ciencia y la tecnología, como práctica social, está orientada hacia fines "de diversa complejidad social (propios del investigador, de la organización y de quienes financian sus trabajos, entre otros) y como tal constituye un conjunto de acciones intencionales" (Albornoz, 2007: 63). A partir de la apertura de algunos puntos en la aplicación de un caso, pretendimos comenzar a entrever el número variado de actores e intereses que implica la puesta en ejecución de una política científica. Asimismo, identificamos algunos de sus principales modificaciones en el tiempo. Advertimos que la misma no se encuentra cerrada, sino que actualmente se encuentra en tensión y discusión el cumplimiento de dicho convenio y sus compromisos enunciados por cada uno de los agentes involucrados. Asimismo, este ensayo se encuentra abierto para seguir pensando cómo se aplicó esta política en otros puntos del país, sus particularidades y diálogos con el caso presentado.

\section{Notas}

(1) Programa completo disponible en http://convocatorias.conicet.gov.ar/vacancia-geografica/ 


\section{Bibliografía}

Albornoz, M. (abril de 2007). Los problemas de la ciencia y el poder. Revista CTS, 3(8), pp. 47-65.

Albornoz, M. y Gordon, A. (2011). La política de ciencia y tecnología en Argentina desde la recuperación de la democracia (1983-2009). En Albornoz, M. y Sebastián, J. (Eds.). Trayectorias de las políticas científicas y universitarias de Argentina y España. Madrid: CSIC.

Elzinga, A. y Jamison, A. (1996). El cambio de las agendas políticas en ciencia y tecnología. Revista Zona Abierta, 75-76, Madrid.

Oszlak, O. y O'Donell, G. (septiembre de 1995). Estado y políticas estatales en América Latina: hacia una estrategia de investigación. Revista REDES, II(4), Unqui, Buenos Aires. 